\title{
Gravitational waveforms for high spin and high mass-ratio binary black holes: A synergistic use of numerical-relativity codes
}

\author{
Ian Hinder, ${ }^{1, *}$ Serguei Ossokine, ${ }^{1, \dagger}$ Harald P. Pfeiffer,,${ }^{1,2, \ddagger}$ and Alessandra Buonanno ${ }^{1,3,}, \S$ \\ ${ }^{1}$ Max Planck Institute for Gravitational Physics (Albert Einstein Institute), Am Mühlenberg 1, Potsdam 14476, Germany \\ ${ }^{2}$ Canadian Institute for Theoretical Astrophysics, University of Toronto, Toronto M5S 3H8, Canada \\ ${ }^{3}$ Department of Physics, University of Maryland, College Park, MD 20742, USA
}

(Dated: October 26, 2018)

\begin{abstract}
Observation and characterisation of gravitational waves from binary black holes requires accurate knowledge of the expected waveforms. The late inspiral and merger phase of the waveform is obtained through direct numerical integration of the full 3-dimensional Einstein equations. The Spectral Einstein Code (SpEC) utilizes a multi-domain pseudo-spectral method tightly adapted to the geometry of the black holes; it is computationally efficient and accurate, but — for high mass-ratios and large spins - sometimes requires manual fine-tuning for the merger-phase of binaries. The Einstein Toolkit (ET) employs finite difference methods and the moving puncture technique; it is less computationally efficient, but highly robust. For some mergers with high mass ratio and large spins, the efficient numerical algorithms used in SpEC have failed, whereas the simpler algorithms used in the ET were successful. Given the urgent need of testing the accuracy of waveform models currently used in LIGO and Virgo inference analyses for high mass ratios and spins, we present here a synergistic approach to numericalrelativity: We combine SpEC and ET waveforms into complete inspiral-merger-ringdown waveforms, taking advantage of the computational efficiency of the pseudo-spectral code during the inspiral, and the robustness of the finite-difference code at the merger. We validate our method against a case where complete waveforms from both codes are available, compute three new hybrid numerical-relativity waveforms, and compare them with analytical waveform models currently used in LIGO and Virgo science. All the waveforms and the hybridization code are publicly available.
\end{abstract}

Introduction. In 2015, Advanced LIGO [1, 2] detected the gravitational wave (GW) event GW150914 corresponding to the merger of a binary black hole (BBH) system [3]. Subsequently, five further GW observations from $\mathrm{BBH}$ and binary neutron-star mergers have been reported [4-8], with the Advanced Virgo detector [9] participating in the more recent observations [6, 7]. The parameters of the BBHs were inferred $[4-8,10,11]$ using fast, analytical waveform models [12-17] calibrated to numerical relativity (NR) simulations [18-22]. The latter are obtained by directly solving the fully nonlinear Einstein equations for BBH spacetimes [23]. The detection efficiency and the accuracy of parameter estimation are directly affected by the accuracy of both analytical and numerical waveform models [24].

The first complete $\mathrm{BBH}$ simulation was obtained by Pretorius in 2005 [25] using finite difference methods and BH excision. The finite difference moving puncture method, which does not require a complex excision algorithm, was developed independently by two groups in late 2005 [26, 27]; because of its simplicity and robustness, this method is in widespread use. Finally, the Spectral Einstein Code [28] (SpEC) makes use of accurate pseudospectral collocation methods combined with BH excision. SpEC improved on existing finite difference solutions in accuracy by one to two orders of magnitude $[29,30]$ and roughly doubled the number of GW cycles in large waveform catalogs [20, 22]. SpEC has performed the longest $\mathrm{BBH}$ simulation to date $(350 \mathrm{GW}$ cycles) [31], as well as the simulations with the largest BH spins (spin-magnitudes 0.998) [32]. The largest mass rato simulations, however, have been performed using moving puncture finite difference codes [21, 33].

Numerical simulations are computationally expensive, typically running for months on large supercomputers. The computational cost to achieve a given accuracy increases with (i) number of orbits $N_{\text {orbs }}$, (ii) the ratio of the masses of the binaries $q=m_{1} / m_{2} \geq 1$, and (iii) increasing magnitudes of the $\mathrm{BH}$ spins. So far, analytic waveform models for BBHs used in LIGO and Virgo follow-up analyses [12-17] have been calibrated to NR waveforms for mild mass ratios and spin magnitudes (e.g., Fig. 1 in [16]), using NR waveforms of $\sim 8-30$ orbits, depending on the binary's parameters and the NR code. The most extreme massratio simulations with spins were presented in [21]. Validation of waveform models for the earlier inspiral and for $\mathrm{BBH}$ parameters too extreme for NR simulations have relied on internal consistency tests (e.g., Ref. [34] for nonspinning BBHs), comparison of twobody dynamics in absence of NR-calibration [35-38], and comparisons between NR-calibrated waveform models with common assumptions and input data ${ }^{1}$. Thus, the true accuracy of waveform models in regions of the parameter space without NR simulations is unknown.

Particularly important are mass ratios $q \gtrsim 4$, and $\mathrm{BH}$ with a large spin-projection along the orbital angular momentum, $\chi_{1,2} \equiv$ $\left(\mathbf{S}_{1,2} \cdot \hat{\mathbf{L}}\right) / m_{1,2}^{2} \gtrsim 0.8$ (e.g., Fig. 1 in [16]). These cases reach particularly high orbital frequency near merger and waveform models needed [16, 22] (or will need [39]) recalibration to new NR waveforms when these became Only accurate NR waveforms in this region of parameter space will ensure that GW parameter measurements in upcoming LIGO and Virgo observations are free of biases due to errors in the waveform models.

Unfortunately, this region of parameter space is computationally very challenging. Resolving the multiple length and time scales associated with both the large and small $\mathrm{BH}$ - made even smaller and more distorted by its high spin - requires high resolution with consequent high computational cost. BH horizons near merger become also particularly distorted complicating $\mathrm{BH}$ excision in the SpEC code, so that BBH inspiral simulations with spins $\chi_{1}=\chi_{2}=0.9$ at mass-ratios $q=3,4,5$ through merger fail shortly before merger.

\footnotetext{
1 Certain NR waveforms are used in the calibration of both waveform models from Refs. [15, 16]; moreover, an uncalibrated effective-one-body model [14], which differs from the one underlying SEOBNRv4 for a few higher-order PN terms, is used during the construction of IMRPhenomD.
} 
In contrast, finite difference codes are able to evolve BBHs with more extreme mass-ratios and spins through merger, albeit only starting with a higher orbital frequency (i.e. lower separation, and covering fewer orbits).

The higher initial frequency implies a significantly higher minimum total mass, $M=m_{1}+m_{2}$, for which the waveform still covers the entire LIGO frequency band. While these shorter waveforms allow calibrating waveform models near merger, the extra low frequency information of longer SpEC simulations may be essential to achieve faithful models and avoid biases in parameter estimation and tests of general relativity from detected GW signals.

Whilst we are confident that the problems with SpEC will be solved by improved numerical methods, the upcoming LIGO and Virgo runs, which promise several tens of GW events from BBHs [40], increase the urgency for accurate waveforms from this region of the parameter space. Thus, we explore here a synergistic approach that combines highly accurate SpEC inspiral evolutions with shorter merger waveforms from finite difference codes (in this case, the Einstein Toolkit $[41,42]$ ) to produce $N R$ $N R$ hybrid waveforms. In this first study, we restrict to quasicircular systems with BH spins parallel or anti-parallel to the orbital angular momentum. This choice simplifies the joining of separate waveforms, because the time shift and spatial rotation required to align waveforms from two simulations starting at different binary separations can be determined in post-processing of the simulation data [43, 44], whereas more generic systems require a more sophisticated treatment, probably involving running additional simulations. After validating our approach, we produce three NR-NR hybrid waveforms for high mass ratios and spin magnitudes, and use them to assess the accuracy of two waveform models currently used in LIGO and Virgo science, namely the spinning effective-one-body waveform model calibrated to NR called SEOBNRv4 [16], and the inspiral-merger-ringdown phenomenological model called IMRPhenomD [15].

In the remainder of this article, we describe the ET and SpEC codes in more detail, explain and validate our hybridization procedure to construct NR-NR hybrid waveforms and then compare the newly constructed waveforms to existing waveform models. Throughout the paper, we use units in which $G=c=1$.

Numerical-relativity codes and waveforms. SpEC [19, 28, 45, 46] is a pseudo-spectral code for efficiently solving partial differential equations, with the primary goal of modeling compactobject binaries. SpEC evolves the first-order formulation [47] of Einstein's equations in generalized-harmonic gauge [48, 49] and employs BH excision [50, 51]. For more details see [52-58].

The Einstein Toolkit [41] is a collection of open source NR components built around the Cactus framework [59]. Our BBH simulations are based on 8th order finite-differencing and the moving puncture method to solve the BSSN [60-62] or CCZ4 [63, 64] formulations of the Einstein equations for Bowen-York initial data $[65,66]$. More details of the specific components and techniques used in the ET can be found in [26, 27, 53, 67-77].

SpEC achieves high accuracy through pseudo-spectral methods, grids tightly adapted to the shape of the binary and use of a computational grid which rotates along with the binary. The $\mathrm{ET}$, in contrast, uses finite-difference methods and straightforward box-in-box mesh refinement with a global inertial computational grid. However, the $\mathrm{SpEC}$ excision algorithm requires accurate and careful tracking of the $\mathrm{BH}$ horizons, particularly for high-spin BHs, to preserve purely-outgoing excision boundary conditions [50]. The high accuracy requirement, combined with strongly deformed BH horizons reduce SpEC's robustness in previously unexplored regions of parameter space, specifically for higher mass-ratio and simultaneous high spins. In contrast, the finite difference moving puncture method (see Refs. [78, 79] for a detailed study) requires no special treatment of the BHs, beyond the choice of suitable coordinate conditions and the requirement to have sufficient grid resolution near them. While finite difference moving puncture evolutions have proven to be extremely robust, great care is needed in the choice of grid-structure to achieve high accuracy.

The NR simulations used in this work are detailed in Table I, where we label cases with $\left(q, \chi_{1}, \chi_{2}\right)$, and where for each case an ET and a SpEC simulation is available. At present, we consider only aligned-spin simulations, so that $\chi_{1,2}$ represent both the spin-projection onto $\mathbf{L}$ as well as the spin-magnitude. The SpEC simulation for case $(3,0.85,0.85)$ completed successfully, including merger and ringdown. It is available in the SXS waveform catalog [80] under ID SXS:BBH:0293 and was first presented in Ref. [81]. The remaining SpEC simulations are new, start $\sim 20$ orbits before merger, but fail around the merger, leading to incomplete waveforms. The ET simulations are all new; their length was chosen to be relatively short, but long enough to overlap with the SpEC-inspirals. All simulations are run at multiple numerical resolutions in order to assess numerical truncation error.

The initial data parameters $\left(q, \chi_{1}, \chi_{2}\right)$ of the ET simulations are identical to those of the SpEC simulations, but due to uncontrolled emission of energy and angular momentum from the initial data slice [82], the relaxed values after the initial dynamical phase deviate slightly from those of SpEC. Table I presents these relaxed values, which differ by $\lesssim 2 \times 10^{-3}$ between the two codes.

Some of the ET evolutions became unstable during ringdown when evolved with the BSSN formulation. Presumably, this instability is related to the high spin of the remnant $\mathrm{BH}, 0.93$, the highest spin $\mathrm{BH}$ we have evolved using the ET code. Ref. [83] reports significant constraint violations in BSSN-evolutions of high spin BHs, which could be mitigated by use of the CCZ4 formulation. Consequently, we re-ran the failing simulations with CCZ4 and constraint-damping parameters $\kappa_{1}=0.1, \kappa_{2}=0$. The resulting evolutions are stable, but needed higher resolution during the inspiral to maintain orbital-phase accuracy.

$N R-N R$ hybrid waveforms and their application. We aim to join an inspiral waveform $h_{\text {insp }}$ and a merger waveform $h_{\text {merger }}$ representing the same astrophysical BBHs, where $h_{\text {insp }}$ terminates shortly before the merger peak, and $h_{\text {merger }}$ overlaps $h_{\text {insp }}$ during the late inspiral. Because the simulations start at different initial orbital separations, $h_{\text {insp }}$ and $h_{\text {merger }}$ will in general differ by a time translation and a spatial rotation. We restrict here to a single spinweight -2 spherical harmonic $\ell=2, m=2$ multipole, and for notational convenience write $h \equiv h_{22} \equiv A e^{i \phi}$, where the second equivalence defines a decomposition in amplitude and phase.

To construct the NR-NR hybrid waveform, we must first determine the time and phase shifts between $h_{\text {merger }}$ and $h_{\text {insp. }}$. We determine $\Delta t$ and $\Delta \phi$ from a portion of the waveform in an interval $\left[t_{1}, t_{2}\right]=\left[t_{2}-T, t_{2}\right]$ where $t_{2}$ is the time at which $h_{i}$ terminates and, say $T=150 \mathrm{M}$, where all times are measured in the coordinate system of $h_{\text {insp }}$. We do not utilize the portion of $h_{\text {merger }}$ for $t<t_{1}$. For quasi-circular BBH systems with spins aligned with the orbital angular momentum, the instanta- 


\begin{tabular}{llllllllllll}
\hline \hline ID & $q^{(S)}$ & $q^{(E)}$ & $\chi_{1}^{(S)}$ & $\chi_{1}^{(E)}$ & $\chi_{2}^{(S)}$ & $\chi_{2}^{(E)}$ & $e^{(S)}$ & $e^{(E)}$ & $N_{\text {orbs }}^{(E)}$ & $M_{\min }^{(S)} / M_{\odot}$ & $M_{\min }^{(E)} / M_{\odot}$ \\
\hline$(3,0.85,0.85)$ & 2.9992 & 2.9986 & 0.850 & 0.851 & 0.849 & 0.851 & $1 \times 10^{-4}$ & $2 \times 10^{-3}$ & 8.0 & 140.9 & 279.4 \\
$(3,0.9,0.9)$ & 2.9988 & 2.9979 & 0.900 & 0.902 & 0.899 & 0.901 & $7 \times 10^{-4}$ & $1 \times 10^{-3}$ & 8.0 & 180.6 & 284.5 \\
$(4,0.9,0.9)$ & 3.9980 & 3.9969 & 0.900 & 0.902 & 0.899 & 0.901 & $9 \times 10^{-4}$ & $6 \times 10^{-4}$ & 7.5 & 178.3 & 322.3 \\
$(5,0.9,0.9)$ & 4.9974 & 4.9955 & 0.900 & 0.902 & 0.899 & 0.901 & $1 \times 10^{-3}$ & $8 \times 10^{-4}$ & 8.5 & 190.4 & 321.9 \\
\hline \hline
\end{tabular}

TABLE I. Properties of the NR waveforms. Shown are the case ID, the mass ratio $q=m_{1} / m_{2} \geq 1$, the dimensionless spin $\chi_{i}$ of each BH $(i=1$ or 2 ) in the direction parallel to the orbital angular momentum, the eccentricity $e$ near the start of the waveform, the number of orbits $N_{\text {orbs }}$, the minimum masses $M_{\min }$ for which the waveform is entirely in the detector band at $10 \mathrm{~Hz}$. Superscripts indicate SpEC (S) and ET (E).

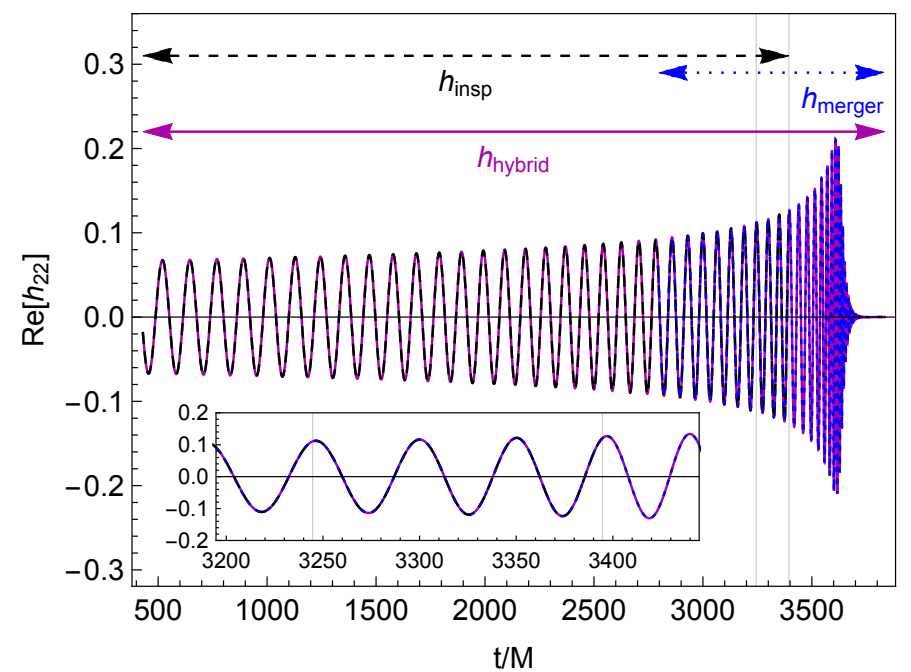

FIG. 1. Gravitational-wave strain. Shown is the real part of the dominant $\ell=2, m=2$ multipole for the case $(5,0.9,0.9)$ from the SpEC inspiral waveform $h_{\text {insp }}$, the ET merger waveform $h_{\text {merger }}$, and the NR-NR hybrid waveform $h_{\text {hybrid }}$ as a function of retarded time $t$ in units of the total mass $M$ of the binary. The vertical lines indicate the hybridisation interval $\left[t_{1}, t_{2}\right]$, which is enlarged in the inset.

neous GW frequency $\omega \equiv d \phi / d t$ increases monotonically with time. We first align $\omega$ in time, which is independent of $\Delta \phi$, and then align the phases. All alignments are performed using leastsquares minimisation. We conclude the alignment by redefining $h_{\text {merger }}(t) \leftarrow e^{i \Delta \phi} h_{\text {merger }}(t+\Delta t)$. We verified that varying the hybridisation interval introduces a negligible error (i.e., a mismatch, as defined below, of less than $10^{-4}$ ). To achieve a smooth transition between $h_{\text {insp }}$ and $h_{\text {merger }}$, we blend $\phi_{\text {insp }}$ with $\phi_{\text {merger }}$ and $A_{\text {insp }}$ with $A_{\text {merger }}$ in the interval $\left[t_{1}, t_{2}\right]$ using a variant of the Planck taper function [84], $\mathcal{T}\left(t ; t_{1}, t_{2}\right)=0$ for $t \leq t_{1}, 1 /\left\{\exp \left[\left(t_{2}-t_{1}\right) /\left(t-t_{1}\right)+\left(t_{2}-t_{1}\right) /\left(t-t_{2}\right)\right]+1\right\}$ for $t_{1}<t<t_{2}$ and 1 for $t \geq t_{2}$. Specifically, we construct $\phi_{\text {hybrid }}=$ $(1-\alpha) \phi_{\text {insp }}+\alpha \phi_{\text {merger }}, A_{\text {hybrid }}=(1-\alpha) A_{\text {insp }}+\alpha A_{\text {merger }}$ where $\alpha(t)=\mathcal{T}\left(t ; t_{1}, t_{2}\right)$. Finally, we construct $h_{\text {hybrid }}=A_{\text {hybrid }} e^{i \phi_{\text {hybrid }}}$.

Figure 1 shows the three waveforms $h_{\text {insp }}, h_{\text {merger }}$ and $h_{\text {hybrid. }}$. There is no visible discrepancy between the inspiral, merger and hybrid waveforms in the hybridisation interval. In fact, $\mid \phi_{\text {merger }}-$ $\phi_{\text {insp }} \mid<6 \times 10^{-3}$ and $\left|1-A_{\text {merger }} / A_{\text {insp }}\right|<4 \times 10^{-4}$ across the hybridisation interval.

NR waveforms are approximations to the solutions of the Einstein equations, and it is necessary to quantify their uncertainty. We consider the impact of truncation error and extrapolation error on the original inspiral and merger waveforms. Truncation error results from using a nonzero grid spacing in the numerical method. Extrapolation error arises from the method used to esti-

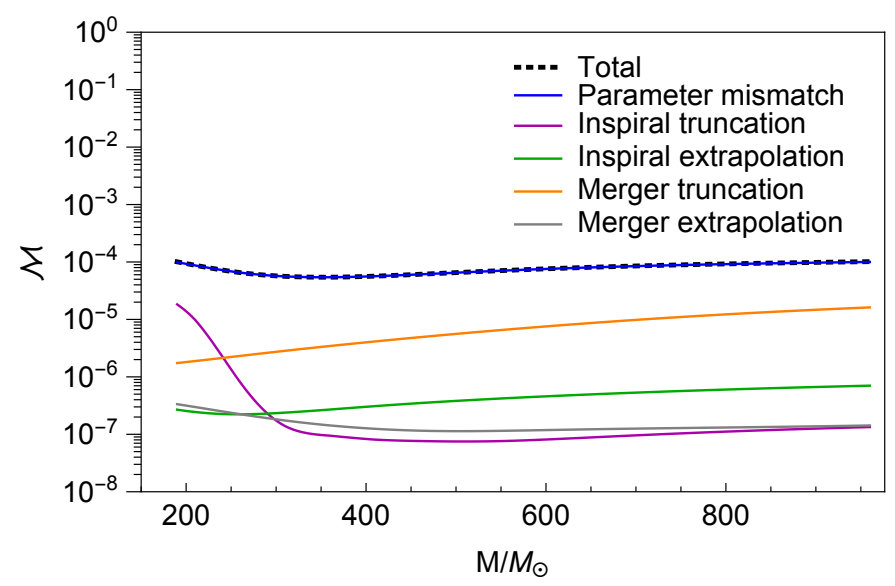

FIG. 2. Estimated uncertainties in the NR-NR hybrid waveform arising from different error sources, expressed in terms of mismatch $\mathcal{M}$ for different total binary masses $M$. Shown is case $(5,0.9,0.9)$.

mate the asymtpotic GWs (at future null infinity) from the GWs extracted at finite-radius. Moreover, an additional error arises because the merger simulation represents a slightly different physical system than the inspiral simulation (as described above). We will see that this is actually the dominant source of error in the NR-NR hybrid waveform.

Gravitational-wave signals from BBHs are often compared using an optimised detector-noise-weighted inner product. Given two waveforms $h_{1}(t)$ and $h_{2}(t)$, their noise-weighted overlap is defined as [85] $\left(h_{1} \mid h_{2}\right) \equiv 4 \operatorname{Re} \int_{f_{\min }}^{f_{\max }} \tilde{h}_{1}(f) \tilde{h}_{2}^{*}(f) / S_{n}(f) \mathrm{d} f$, where $\tilde{h}_{1,2}(f)$ are the Fourier transforms of the waveforms and $S_{n}(f)$ is the one-sided noise power spectral density, chosen here as the zero-detuned-high-power variant from [86]. We use $f_{\min }=$ $10 \mathrm{~Hz}, f_{\max }=8192 \mathrm{~Hz}$. The match between two waveforms is then defined as the overlap between the normalized waveforms maximized over relative time and phase shifts, $M\left(h_{1}, h_{2}\right) \equiv$ $\max _{\phi_{c}, t_{c}}\left(h_{1}\left(\phi_{c}, t_{c}\right) \mid h_{2}\right) / \sqrt{\left(h_{1} \mid h_{1}\right)\left(h_{2} \mid h_{2}\right)}$. The match measures how similar the waveforms appear to a GW detector when the data is analyzed using matched filtering. For convenience, since the differences we consider are small, we consider the mismatch $\mathcal{M}\left(h_{1}, h_{2}\right) \equiv 1-M\left(h_{1}, h_{2}\right)$. A mismatch of zero indicates that the waveforms appear identical.

Figure 2 summarizes the error budget for an NR-NR hybrid for the case $(5,0.9,0.9)$ as a function of the total mass $M$ of the binary (a single NR BBH simulation can represent a binary of any total mass by a scaling of the waveform). Each curve represents the mismatch between a NR-NR hybrid waveform and a perturbed NR-NR hybrid. For the inspiral and merger truncation error curves, the perturbed hybrid is constructed using a lower resolution inspiral and merger waveform, respectively. Similarly, for 


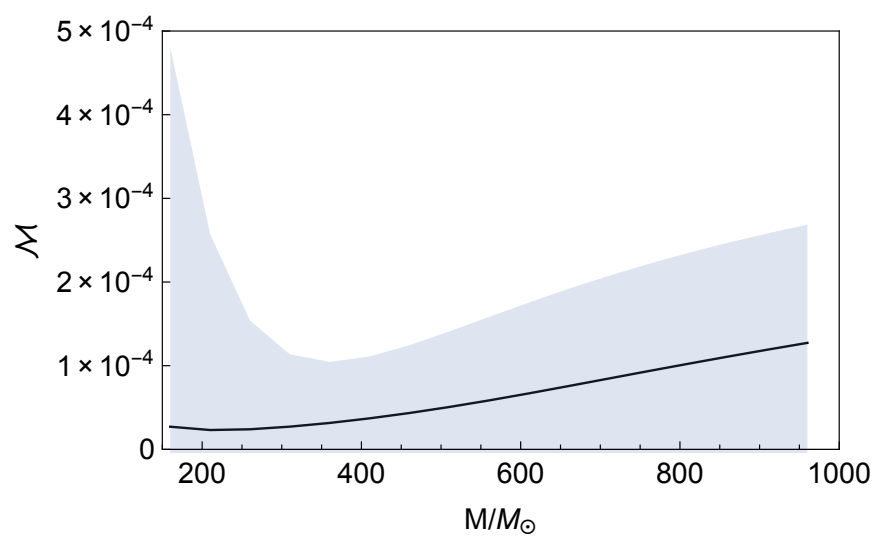

FIG. 3. Mismatch $\mathcal{M}$ between SpEC-ET hybrid and complete SpEC waveforms for the case $(3,0.85,0.85)$ where the SpEC waveform includes a merger. The shaded region represents the estimated uncertainty in this computation coming from errors in the SpEC and ET waveforms.

the inspiral and merger extrapolation-error curves, each perturbed hybrid is constructed using higher-order extrapolation for the inspiral or merger waveforms, respectively. Furthermore, the parameter mismatch curve compares a standard SEOBNRv4 waveform [16] with parameters of the inspiral simulation with a hybrid waveform constructed in the same way as the NR-NR hybrid but from two SEOBNRv4 waveforms, the first using the parameters of the inspiral simulation, and the second using the parameters of the merger simulation. We assume that the mismatch introduced by hybridising two SEOBNRv4 waveforms with different parameters is representative of the same effect with NR waveforms.

The total error budget in Fig. 2 is the quadrature-sum of the individual contributions, and provides a conservative indication of the total uncertainty in the waveform as seen by a GW detector. Figure 2 demonstrates that the error of the hybrid waveform is dominated by the slightly inconsistent $\mathrm{BBH}$ parameters for $h_{\text {insp }}$ and $h_{\text {merger }}$. This is also the case for all the other NR configurations. These parameter inconsistencies could be reduced by repeating the merger simulation with initial mass ratios and spins adjusted in such a way that the relaxed values approach those of the inspiral simulation. In the future, this step should be performed at the same time as the iterative eccentricity reduction, which already requires multiple simulations.

Figure 3 tests our hybridisation method and our error estimates for the case $(3,0.85,0.85)$, for which complete inspiral-mergerringdown SpEC-waveforms are available. Discarding the SpEC merger, we construct a SpEC-ET hybrid waveform as described above, and plot its mismatch with the complete SpEC waveform as the solid black line. The grey band in Fig. 3 represents our error-estimate on the hybrid, computed as the quadrature sum of the effects on the mismatch between $h_{\text {hybrid }}$ and $h_{\text {insp }}$ of perturbing the input waveforms by their individual sources of error. Figure 2 indicates that the hybridization introduces only small errors $(\mathcal{M} \lesssim$ $10^{-4}$ ), and confirms the validity of our error estimate from Fig. 2.

We construct NR-NR hybrid waveforms for each of the configurations shown in Table I, with properties of these NR-NR hybrids given in Table II. All cases have errors comparable to the validation case $(3,0.85,0.85)$ shown in Fig. 2 . In each case, the dominant source of error is the parameter mismatch between the inspiral and merger simulations. The maximum estimated uncertainty in the NR-NR hybrid across all the configurations is a mismatch of $1 \times 10^{-4}$.

It is not in general known how the mismatch between an approximate waveform and the true waveform relates to biases in parameter recovery except in specific configurations which have been studied. Ref. [24] found that a waveform mismatch of $\sim 5 \times 10^{-3}$ did not lead to noticeable parameter estimation biases for binaries comparable to GW $150914^{2}$, though it is not clear how to translate this result to the more extreme configurations we study here. Moreover, differences between the analytical waveform models considered in the next section and our NR-NR hybrids are $\sim 10$-times larger than $\mathcal{M}_{\text {hybrid }}^{\delta}$ reported in Table II. The waveform models SEOBNRv4 and IMRPhenomD were found to be accurate to NR waveforms to a mismatch of $\mathcal{M} \lesssim 10^{-2}$, so the NR-NR hybrid waveforms are an order of magnitude more accurate, and hence suitable for comparing with these waveform models. Finally, the distinguishability criterion [87-90], $\mathcal{M}<D /\left(2 \rho^{2}\right)$ where $\rho$ is the SNR of the signal and $D$ is the number of intrinsic parameters that would not be measured accurately due to inaccuracy of the waveform model. For aligned-spin systems considered in this paper we take $D=4$, and using the largest mismatch from all the configurations, we find that the waveforms presented here would be indistinguishable by Advanced LIGO for signal-to-noise ratios $\lesssim 140$, which includes most of the expected detectable events. We are therefore satisfied that the NR-NR hybrid waveforms are sufficiently accurate for use in waveform modelling.

As a first application of our synergistic approach of combining NR codes to compute waveforms for large mass ratios and spins, we compare the new NR-NR hybrid waveforms with the analytical waveform models SEOBNRv4 and IMRPhenomD currently employed in LIGO and Virgo science. We evaluate these waveform models at the relaxed values of mass ratio and spin computed from the SpEC simulations in Table I.

Figure 4 shows the mismatch between the NR-NR hybrid and the waveform models for each case. The very thin, shaded regions around each curve indicate the estimated error of the NRNR hybrid, computed as the quadrature sum of the effects on the mismatch between $h_{\text {hybrid }}$ and the model waveforms of perturbing the $h_{\text {hybrid }}$ by their individual sources of error. These errors in the hybrids themselves are negligible in comparison with the difference between the NR-NR hybrids and the waveform models. The curves start at the minimum masses for which the NR waveforms reach down to $10 \mathrm{~Hz}$, and hence cover the frequency range of Advanced LIGO and Virgo at design sensitivity. Comparisons would only be possible at significantly higher total mass if only the shorter ET waveforms were used.

\begin{tabular}{lll}
\hline \hline ID & $N_{\text {orbs }}^{(H)}$ & $\mathcal{M}_{\text {hybrid }}^{\delta}$ \\
\hline$(3,0.85,0.85)$ & 23.9 & $3 \times 10^{-5}$ \\
$(3,0.9,0.9)$ & 16.3 & $7 \times 10^{-5}$ \\
$(4,0.9,0.9)$ & 19.3 & $1 \times 10^{-4}$ \\
$(5,0.9,0.9)$ & 20.0 & $1 \times 10^{-4}$ \\
\hline \hline
\end{tabular}

TABLE II. Properties of the NR-NR hybrid waveforms. Shown are the case ID, the number of orbits $N_{\text {orbs }}$, and the estimated uncertainty $\mathcal{M}_{\text {hybrid }}^{\delta}$ on the hybrid, computed as described in the text.

\footnotetext{
${ }^{2}$ Except for nearly edge-on systems where errors are dominated by the lack of sub-dominant modes in the waveform models.
} 


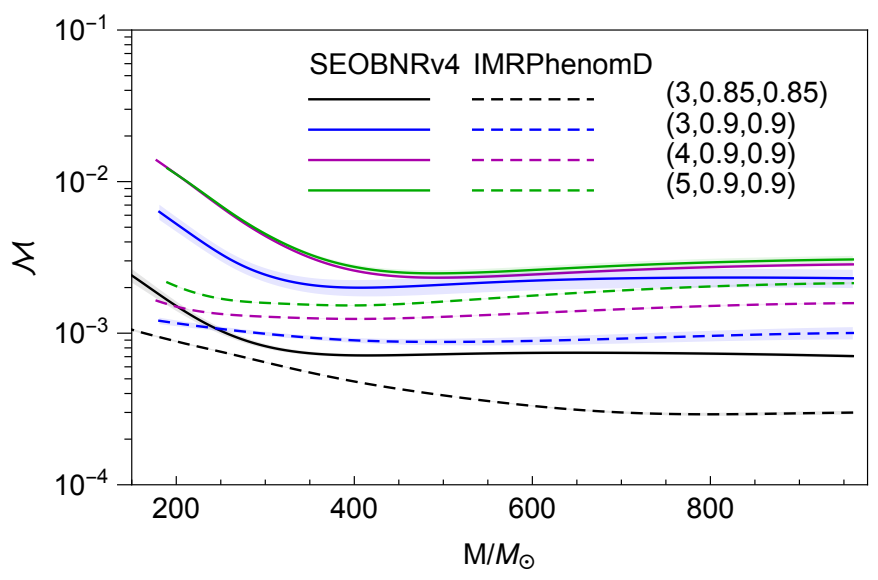

FIG. 4. Mismatch $\mathcal{M}$ between SpEC-ET hybrids and the waveform models SEOBNRv4 (solid lines) and IMRPhenomD (dashed lines) for all the configurations in Table I.

The waveform models had mismatches of typically $\lesssim 10^{-2}$ with the NR waveforms they were originally tested (or calibrated) against. We see that for the more extreme configurations presented here, both waveform models maintain this accuracy. The massrange $M \gtrsim 200 M_{\odot}$ of the comparison in Fig. 4 is determined by the requirement that the NR-NR hybrid waveforms cover the entire Advanced LIGO sensitivity band. Waveforms with lower initial orbital frequency (i.e. more orbits) are required to extend this calculation to lower mass. The NR waveforms produced here could now be used to improve the waveform models, if more accuracy in this region of the parameter space is required, for example for future GW detectors with higher sensitivities.

Conclusions. BBH simulations for systems with both high mass ratio and high spin remain challenging for NR codes, and yet it is in this region of the parameter space where waveform models typically depend strongly on calibration to NR simulations $[15,16]$. The SpEC code, based on accurate pseudo-spectral methods, sophisticated adapted grids and corotating coordinates has a long history of producing large numbers of long ( $~ 225$ orbits) simulations (e.g., see Refs. [20, 81]), but sometimes requires significant fine-tuning in order to simulate the merger [51, 91]. Finite difference codes, on the other hand, have generally been used to produce shorter simulations, typically $\lesssim 15$ orbits (see, e.g., Refs. [21, 92, 93]), possibly reflecting a lower accuracy for a given computational cost, due to the use of more straightforward numerical methods. However, maybe as a result of this simplicity, these codes are able to handle some more extreme BBH configurations where SpEC currently fails to simulate the merger.

We have taken advantage of the strengths of both codes to combine accurate and efficient SpEC inspirals with robust finite difference mergers, and have expanded the parameter space of available NR waveforms. With the resulting new NR hybrid waveforms, we test the state-of-the-art waveform models SEOBNRv4 and IMRPhenomD. We find that the models agree with the new NR waveforms to mismatch $\mathcal{M} \lesssim 1 \%$, for total binary masses for which the entire NR-NR hybrid waveform is in the Advanced LIGO detector band starting at $10 \mathrm{~Hz}$. This is sufficient accuracy for current waveform modelling purposes.

While we anticipate that the problems encountered at the merger with SpEC will be resolved with improved numerical methods, our synergistic hybridisation approach provides a use- ful stop-gap measure to extend the science reach of current NR codes, and hence the parameter space of waveforms available for waveform-model validation for upcoming $\mathrm{GW}$ science with LIGO and Virgo detectors. The method is completely generic for aligned-spin binaries and is applicable to any pair of NR codes. A useful next step would be to apply the method to the subdominant modes $(\ell>2)$, as these are important for systems with the high mass ratios studied here. For precessing binaries, however, it requires techniques to match the precessing spin-directions between the two waveforms at a certain reference frequency.

All the waveforms presented here, including the NR-NR hybrids, are publicly available [94]. An implementation of the hybrid construction method is available in the SimulationTools for Mathematica [77] package, and a Mathematica notebook demonstrating the method is provided in [94].

Acknowledgments. Calculations were performed using the Spectral Einstein code (SpEC) [28] and the Einstein Toolkit [42] on the Minerva high-performance computer cluster at the Max Planck Institute for Gravitational Physics in Potsdam. The Einstein Toolkit simulations were originally based on the Einstein Toolkit GW150914 BBH example [76]. Analysis of the numerical data was performed using SimulationTools for Mathematica [77]. H.P. gratefully acknowledges funding from NSERC of Canada, the Ontario Early Researcher Awards Program, and the Canada Research Chairs Program.

* ian.hinder@aei.mpg.de

$\dagger$ serguei.ossokine@aei.mpg.de

¥ harald.pfeiffer@aei.mpg.de

$\S$ alessandra.buonanno@aei.mpg.de

[1] J. Aasi et al. (LIGO Scientific), Class. Quant. Grav. 32, 074001 (2015), arXiv:1411.4547 [gr-qc].

[2] B. P. Abbott et al. (Virgo, LIGO Scientific), Phys. Rev. Lett. 116, 131103 (2016), arXiv:1602.03838 [gr-qc].

[3] B. P. Abbott et al. (Virgo, LIGO Scientific), Phys. Rev. Lett. 116, 061102 (2016), arXiv:1602.03837 [gr-qc].

[4] B. P. Abbott et al. (Virgo, LIGO Scientific), Phys. Rev. Lett. 116, 241103 (2016), arXiv:1606.04855 [gr-qc].

[5] B. P. Abbott et al. (VIRGO, LIGO Scientific), Phys. Rev. Lett. 118, 221101 (2017), arXiv:1706.01812 [gr-qc].

[6] B. P. Abbott et al. (Virgo, LIGO Scientific), Phys. Rev. Lett. 119, 141101 (2017), arXiv:1709.09660 [gr-qc].

[7] B. Abbott et al. (Virgo, LIGO Scientific), Phys. Rev. Lett. 119, 161101 (2017), arXiv:1710.05832 [gr-qc].

[8] B. P. Abbott et al. (Virgo, LIGO Scientific), Astrophys. J. 851, L35 (2017), arXiv:1711.05578 [astro-ph.HE].

[9] F. Acernese et al. (VIRGO), Class. Quant. Grav. 32, 024001 (2015), arXiv:1408.3978 [gr-qc].

[10] B. P. Abbott et al. (Virgo, LIGO Scientific), Phys. Rev. Lett. 116, 241102 (2016), arXiv:1602.03840 [gr-qc].

[11] T. D. Abbott et al. (Virgo, LIGO Scientific), Phys. Rev. X6, 041014 (2016), arXiv:1606.01210 [gr-qc].

[12] Y. Pan, A. Buonanno, A. Taracchini, L. E. Kidder, A. H. Mroué, H. P. Pfeiffer, M. A. Scheel, and B. Szilagyi, Phys. Rev. D89, 084006 (2014), arXiv:1307.6232 [gr-qc].

[13] M. Hannam, P. Schmidt, A. Bohe, L. Haegel, S. Husa, F. Ohme, G. Pratten, and M. Purrer, Phys. Rev. Lett. 113, 151101 (2014), arXiv:1308.3271 [gr-qc].

[14] A. Taracchini et al., Phys. Rev. D89, 061502 (2014), arXiv:1311.2544 [gr-qc].

[15] S. Khan, S. Husa, M. Hannam, F. Ohme, M. Pürrer, X. Jiménez Forteza, and A. Bohé, Phys. Rev. D93, 044007 (2016), 
arXiv:1508.07253 [gr-qc].

[16] A. Bohé et al., Phys. Rev. D95, 044028 (2017), arXiv:1611.03703 [gr-qc].

[17] S. Babak, A. Taracchini, and A. Buonanno, Phys. Rev. D95, 024010 (2017), arXiv:1607.05661 [gr-qc].

[18] M. Hannam, S. Husa, F. Ohme, D. Müller, and B. Brügmann, Phys. Rev. D 82, 124008 (2010), arXiv:1007.4789.

[19] L. T. Buchman, H. P. Pfeiffer, M. A. Scheel, and B. Szilagyi, Phys. Rev. D86, 084033 (2012), arXiv:1206.3015 [gr-qc].

[20] A. H. Mroue et al., Phys. Rev. Lett. 111, 241104 (2013), arXiv:1304.6077 [gr-qc].

[21] S. Husa, S. Khan, M. Hannam, M. Pürrer, F. Ohme, X. Jiménez Forteza, and A. Bohé, Phys. Rev. D93, 044006 (2016), arXiv:1508.07250 [gr-qc].

[22] P. Kumar, T. Chu, H. Fong, H. P. Pfeiffer, M. Boyle, D. A. Hemberger, L. E. Kidder, M. A. Scheel, and B. Szilagyi, Phys. Rev. D93, 104050 (2016), arXiv:1601.05396 [gr-qc].

[23] T. W. Baumgarte and S. L. Shapiro, Numerical Relativity: Solving Einstein's Equations on the Computer (Cambridge University Press, New York, 2010).

[24] B. P. Abbott et al. (Virgo, LIGO Scientific), Class. Quant. Grav. 34, 104002 (2017), arXiv:1611.07531 [gr-qc].

[25] F. Pretorius, Phys. Rev. Lett. 95, 121101 (2005), arXiv:grqc/0507014 [gr-qc].

[26] J. G. Baker, J. Centrella, D.-I. Choi, M. Koppitz, and J. van Meter, Phys. Rev. Lett. 96, 111102 (2006), arXiv:gr-qc/0511103.

[27] M. Campanelli, C. O. Lousto, P. Marronetti, and Y. Zlochower, Phys. Rev. Lett. 96, 111101 (2006), gr-qc/0511048.

[28] http://www.black-holes.org/SpEC.html.

[29] M. Hannam, S. Husa, J. G. Baker, M. Boyle, B. Bruegmann, T. Chu, N. Dorband, F. Herrmann, I. Hinder, B. J. Kelly, L. E. Kidder, P. Laguna, K. D. Matthews, J. R. van Meter, H. P. Pfeiffer, D. Pollney, C. Reisswig, M. A. Scheel, and D. Shoemaker, Phys. Rev. D 79, 084025 (2009), arXiv:0901.2437 [gr-qc].

[30] I. Hinder et al., Class. Quant. Grav. 31, 025012 (2014), arXiv:1307.5307 [gr-qc].

[31] B. Szilágyi, J. Blackman, A. Buonanno, A. Taracchini, H. P. Pfeiffer, M. A. Scheel, T. Chu, L. E. Kidder, and Y. Pan, Phys. Rev. Lett. 115, 031102 (2015), arXiv:1502.04953 [gr-qc].

[32] K. Chatziioannou, G. Lovelace, M. Boyle, M. Giesler, D. A. Hemberger, R. Katebi, L. E. Kidder, H. P. Pfeiffer, M. A. Scheel, and B. Szilágyi, Phys. Rev. D98, 044028 (2018), arXiv:1804.03704 [grqc].

[33] C. O. Lousto and Y. Zlochower, Phys. Rev. Lett. 106, 041101 (2011), arXiv:1009.0292 [gr-qc].

[34] Y. Pan, A. Buonanno, A. Taracchini, M. Boyle, L. E. Kidder, A. H. Mroué, H. P. Pfeiffer, M. A. Scheel, B. Szilágyi, and A. Zenginoglu, Phys. Rev. D89, 061501 (2014), arXiv:1311.2565 [gr-qc].

[35] T. Damour, A. Nagar, D. Pollney, and C. Reisswig, Phys. Rev. Lett. 108, 131101 (2012), arXiv:1110.2938 [gr-qc].

[36] A. Le Tiec, A. H. Mroue, L. Barack, A. Buonanno, H. P. Pfeiffer, N. Sago, and A. Taracchini, Phys. Rev. Lett. 107, 141101 (2011), arXiv:1106.3278 [gr-qc].

[37] A. Le Tiec, E. Barausse, and A. Buonanno, Phys. Rev. Lett. 108, 131103 (2012), arXiv:1111.5609 [gr-qc].

[38] S. Ossokine, T. Dietrich, E. Foley, R. Katebi, and G. Lovelace, (2017), arXiv: 1712.06533 [gr-qc].

[39] A. Nagar et al., (2018), arXiv:1806.01772 [gr-qc].

[40] B. P. Abbott et al. (VIRGO, KAGRA, LIGO Scientific), Living Rev. Rel. 21, 3 (2018), [Living Rev. Rel.19,1(2016)], arXiv:1304.0670 [gr-qc].

[41] F. Loffler et al., Class. Quant. Grav. 29, 115001 (2012), arXiv:1111.3344 [gr-qc].

[42] EinsteinToolkit, "Einstein Toolkit: Open software for relativistic astrophysics,".

[43] Y. Pan, A. Buonanno, J. G. Baker, J. Centrella, B. J. Kelly, S. T. McWilliams, F. Pretorius, and J. R. van Meter, Phys. Rev. D77, 024014 (2008), arXiv:0704.1964 [gr-qc].
[44] P. Ajith et al., Phys. Rev. D77, 104017 (2008), [Erratum: Phys. Rev.D79,129901(2009)], arXiv:0710.2335 [gr-qc].

[45] M. A. Scheel, H. P. Pfeiffer, L. Lindblom, L. E. Kidder, O. Rinne, and S. A. Teukolsky, Phys. Rev. D74, 104006 (2006), arXiv:grqc/0607056 [gr-qc].

[46] B. Szilagyi, L. Lindblom, and M. A. Scheel, Phys. Rev. D80, 124010 (2009), arXiv:0909.3557 [gr-qc].

[47] L. Lindblom, M. A. Scheel, L. E. Kidder, R. Owen, and O. Rinne, Class. Quant. Grav. 23, S447 (2006), arXiv:gr-qc/0512093 [gr-qc].

[48] H. Friedrich, Communications in Mathematical Physics 100, 525 (1985).

[49] F. Pretorius, Phys. Rev. Lett. 95, 121101 (2005), arXiv:grqc/0507014 [gr-qc].

[50] D. A. Hemberger, M. A. Scheel, L. E. Kidder, B. Szilágyi, G. Lovelace, N. W. Taylor, and S. A. Teukolsky, Class. Quant. Grav. 30, 115001 (2013), arXiv:1211.6079 [gr-qc].

[51] M. A. Scheel, M. Giesler, D. A. Hemberger, G. Lovelace, K. Kuper, M. Boyle, B. Szilágyi, and L. E. Kidder, Class. Quant. Grav. 32, 105009 (2015), arXiv:1412.1803 [gr-qc].

[52] L. Lindblom and B. Szilagyi, Phys. Rev. D80, 084019 (2009), arXiv:0904.4873 [gr-qc].

[53] H. P. Pfeiffer, D. A. Brown, L. E. Kidder, L. Lindblom, G. Lovelace, and M. A. Scheel, New frontiers in numerical relativity. Proceedings, International Meeting, NFNR 2006, Potsdam, Germany, July 17-21, 2006, Class. Quant. Grav. 24, S59 (2007), arXiv:grqc/0702106 [gr-qc].

[54] A. Buonanno, L. E. Kidder, A. H. Mroué, H. P. Pfeiffer, and A. Taracchini, Phys. Rev. D 83, 104034 (2011), arXiv:1012.1549 [gr-qc].

[55] B. Szilágyi, Int. J. Mod. Phys. D23, 1430014 (2014), arXiv:1405.3693 [gr-qc].

[56] M. Boyle and A. H. Mroue, Phys. Rev. D80, 124045 (2009), arXiv:0905.3177 [gr-qc].

[57] M. Boyle, Phys. Rev. D87, 104006 (2013), arXiv:1302.2919 [gr-qc].

[58] N. W. Taylor, M. Boyle, C. Reisswig, M. A. Scheel, T. Chu, L. E. Kidder, and B. Szilágyi, Phys. Rev. D88, 124010 (2013), arXiv:1309.3605 [gr-qc].

[59] T. Goodale, G. Allen, G. Lanfermann, J. Massó, T. Radke, E. Seidel, and J. Shalf, in Vector and Parallel Processing - VECPAR'2002, 5th International Conference, Lecture Notes in Computer Science (Springer, Berlin, 2003).

[60] T. Nakamura, K. Oohara, and Y. Kojima, Prog. Theor. Phys. Suppl. 90, 1 (1987).

[61] M. Shibata and T. Nakamura, Phys. Rev. D52, 5428 (1995).

[62] T. W. Baumgarte and S. L. Shapiro, Phys. Rev. D59, 024007 (1999), arXiv:gr-qc/9810065.

[63] C. Bona, T. Ledvinka, C. Palenzuela, and M. Zacek, Phys. Rev. D67, 104005 (2003), arXiv:gr-qc/0302083 [gr-qc].

[64] D. Alic, C. Bona-Casas, C. Bona, L. Rezzolla, and C. Palenzuela, Phys. Rev. D85, 064040 (2012), arXiv:1106.2254 [gr-qc].

[65] J. M. Bowen and J. W. York, Phys. Rev. D 21, 2047 (1980).

[66] S. Brandt and B. Brügmann, Phys. Rev. Lett. 78, 3606 (1997).

[67] M. Ansorg, B. Bruegmann, and W. Tichy, Phys. Rev. D70, 064011 (2004), arXiv:gr-qc/0404056 [gr-qc].

[68] J. D. Brown, P. Diener, O. Sarbach, E. Schnetter, and M. Tiglio, Phys. Rev. D79, 044023 (2009), arXiv:0809.3533 [gr-qc].

[69] E. Schnetter, S. H. Hawley, and I. Hawke, Class. Quant. Grav. 21, 1465 (2004), arXiv:gr-qc/0310042 [gr-qc].

[70] D. Pollney, C. Reisswig, E. Schnetter, N. Dorband, and P. Diener, Phys. Rev. D83, 044045 (2011), arXiv:0910.3803 [gr-qc].

[71] J. Thornburg, Class. Quant. Grav. 21, 743 (2004), arXiv:grqc/0306056 [gr-qc].

[72] O. Dreyer, B. Krishnan, D. Shoemaker, and E. Schnetter, Phys. Rev. D67, 024018 (2003), arXiv:gr-qc/0206008 [gr-qc].

[73] C. Reisswig and D. Pollney, Class. Quantum Grav. 28, 195015 (2011), arXiv:1006.1632 [gr-qc].

[74] S. Husa, I. Hinder, and C. Lechner, Comput. Phys. Commun. 174, 983 (2006), arXiv:gr-qc/0404023. 
[75] M. Thomas and E. Schnetter, in Grid Computing (GRID), 2010 11th IEEE/ACM International Conference on (2010) pp. 369 -378, arXiv:1008.4571 [cs.DC].

[76] B. Wardell, I. Hinder, and E. Bentivegna, "Simulation of GW150914 binary black hole merger using the Einstein Toolkit," (2016).

[77] I. Hinder and B. Wardell, "SimulationTools for Mathematica," http: //simulationtools.org.

[78] M. Hannam, S. Husa, D. Pollney, B. Bruegmann, and N. O’Murchadha, Phys. Rev. Lett. 99, 241102 (2007), arXiv:grqc/0606099 [gr-qc].

[79] M. Hannam, S. Husa, F. Ohme, B. Bruegmann, and N. O’Murchadha, Phys. Rev. D78, 064020 (2008), arXiv:0804.0628 [gr-qc].

[80] https://www.black-holes.org/waveforms/catalog.php.

[81] T. Chu, H. Fong, P. Kumar, H. P. Pfeiffer, M. Boyle, D. A. Hemberger, L. E. Kidder, M. A. Scheel, and B. Szilagyi, Class. Quant. Grav. 33, 165001 (2016), arXiv:1512.06800 [gr-qc].

[82] G. Lovelace, Numerical relativity data analysis. Proceedings, 2nd Meeting, NRDA 2008, Syracuse, USA, August 11-14, 2008, Class. Quant. Grav. 26, 114002 (2009), arXiv:0812.3132 [gr-qc].

[83] Y. Zlochower, J. Healy, C. O. Lousto, and I. Ruchlin, Phys. Rev. D96, 044002 (2017), arXiv:1706.01980 [gr-qc].

[84] D. J. A. McKechan, C. Robinson, and B. S. Sathyaprakash, Gravitational waves. Proceedings, 8th Edoardo Amaldi Conference, Amaldi 8, New York, USA, June 22-26, 2009, Class. Quant. Grav. 27, 084020
(2010), arXiv:1003.2939 [gr-qc].

[85] L. S. Finn, Phys. Rev. D46, 5236 (1992), arXiv:gr-qc/9209010 [grqc].

[86] D. Shoemaker (LIGO Collaboration), "Advanced LIGO anticipated sensitivity curves," (2010), LIGO Document T0900288-v3.

[87] E. E. Flanagan and S. A. Hughes, Phys. Rev. D57, 4566 (1998), arXiv:gr-qc/9710129 [gr-qc].

[88] L. Lindblom, B. J. Owen, and D. A. Brown, Phys. Rev. D78, 124020 (2008), arXiv:0809.3844 [gr-qc].

[89] S. T. McWilliams, B. J. Kelly, and J. G. Baker, Phys. Rev. D82, 024014 (2010), arXiv:1004.0961 [gr-qc].

[90] K. Chatziioannou, A. Klein, N. Yunes, and N. Cornish, Phys. Rev. D95, 104004 (2017), arXiv:1703.03967 [gr-qc].

[91] M. A. Scheel, M. Boyle, T. Chu, L. E. Kidder, K. D. Matthews, and H. P. Pfeiffer, Phys. Rev. D79, 024003 (2009), arXiv:0810.1767 [gr-qc].

[92] J. Healy, C. O. Lousto, Y. Zlochower, and M. Campanelli, Class. Quant. Grav. 34, 224001 (2017), arXiv:1703.03423 [gr-qc].

[93] K. Jani, J. Healy, J. A. Clark, L. London, P. Laguna, and D. Shoemaker, Class. Quant. Grav. 33, 204001 (2016), arXiv:1605.03204 [gr-qc].

[94] I. Hinder, S. Ossokine, H. Pfeiffer, and A. Buonanno, "Waveform data for Gravitational waveforms for high spin and high mass-ratio binary black holes: A synergistic use of numerical-relativity codes," (2018). 